\title{
Machine learning enables detection of early-stage colorectal cancer by whole-genome sequencing of plasma cell-free DNA
}

Nathan Wan ${ }^{\dagger}$, David Weinberg ${ }^{\dagger}$, Tzu-Yu Liu, Katherine Niehaus, Eric A. Ariazi, Daniel Delubac, Ajay Kannan, Brandon White, Mitch Bailey, Marvin Bertin, Nathan Boley, Derek Bowen, James Cregg, Adam M. Drake, Riley Ennis, Signe Fransen, Erik Gafni, Loren Hansen, Yaping Liu, Gabriel L. Otte, Jennifer Pecson, Brandon Rice, Gabriel E. Sanderson, Aarushi Sharma, John St. John, Catherina Tang, Abraham Tzou, Leilani Young, Girish Putcha $^{* \dagger}$ and Imran S. Haque ${ }^{\dagger}$

\begin{abstract}
Background: Blood-based methods using cell-free DNA (cfDNA) are under development as an alternative to existing screening tests. However, early-stage detection of cancer using tumor-derived cfDNA has proven challenging because of the small proportion of cfDNA derived from tumor tissue in early-stage disease. A machine learning approach to discover signatures in cfDNA, potentially reflective of both tumor and non-tumor contributions, may represent a promising direction for the early detection of cancer.

Methods: Whole-genome sequencing was performed on cfDNA extracted from plasma samples ( $N=546$ colorectal cancer and 271 non-cancer controls). Reads aligning to protein-coding gene bodies were extracted, and read counts were normalized. cfDNA tumor fraction was estimated using IchorCNA. Machine learning models were trained using kfold cross-validation and confounder-based cross-validations to assess generalization performance.

Results: In a colorectal cancer cohort heavily weighted towards early-stage cancer (80\% stage I/II), we achieved a mean AUC of 0.92 (95\% Cl 0.91-0.93) with a mean sensitivity of 85\% (95\% Cl 83-86\%) at 85\% specificity. Sensitivity generally increased with tumor stage and increasing tumor fraction. Stratification by age, sequencing batch, and institution demonstrated the impact of these confounders and provided a more accurate assessment of generalization performance.

Conclusions: A machine learning approach using cfDNA achieved high sensitivity and specificity in a large, predominantly early-stage, colorectal cancer cohort. The possibility of systematic technical and institution-specific biases warrants similar confounder analyses in other studies. Prospective validation of this machine learning method and evaluation of a multi-analyte approach are underway.
\end{abstract}

Keywords: Cell-free DNA, Colorectal cancer, Screening, Whole-genome sequencing, Early-stage cancer

\footnotetext{
* Correspondence: authors@freenome.com

${ }^{\dagger}$ Nathan Wan and David Weinberg are co-first authors.

${ }^{+}$Girish Putcha and Imran S. Haque are co-senior authors.

Freenome, South San Francisco, CA, USA
}

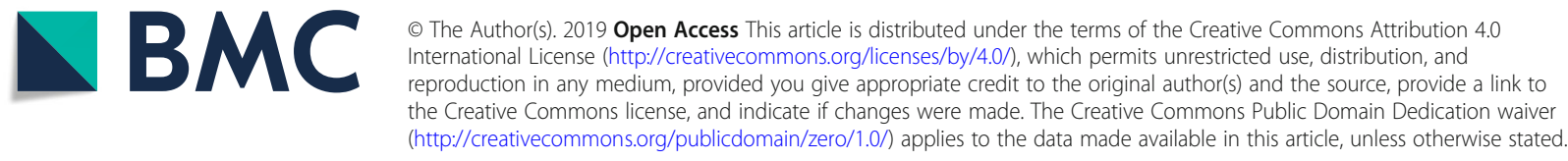




\section{Background}

Despite the public health emphasis on population-level cancer screening in recent decades, adherence remains lower than desired [1], and cancer is often detected too late for successful treatment. For example, nearly $60 \%$ of colorectal cancer (CRC) cases, and approximately $80 \%$ of pancreatic cancer cases, are detected after regional or distant metastases [2]. Although the burden of CRC has been decreasing, CRC remains the third leading cause of cancer-related deaths in men and women in the United States [2]. Current cancer screening methods are often invasive, inconvenient, expensive, and/or have suboptimal clinical performance (i.e., sensitivity or specificity), particularly for early-stage disease and precancerous lesions [3].

Recently, blood-based screening tests for cancer have been proposed in an effort to address some of the aforementioned challenges. One key area of both academic and commercial interest is circulating cell-free DNA (cfDNA), which includes both tumor-derived DNA (socalled "circulating tumor DNA", or ctDNA) and DNA derived from non-tumor cells, such as hematopoietic and stromal cells, to supplement or replace existing cancer screening methods.

Different screening approaches using cfDNA are being explored, and some have hypothesized that ctDNA-only based "liquid biopsies" may enable sensitive and specific early detection of cancer ([4-7]. ctDNA has unique characteristics of tumor DNA, such as cancer-associated mutations, translocations, and/or large chromosomal copy number variants (CNVs), not typically present in the cfDNA of healthy patients [8]. In addition, ctDNA fragments appear to be shorter on average than cfDNA found in healthy subjects [9]. However, others have questioned whether such an approach is feasible for routine screening, given biological (e.g., clonal hematopoiesis of indeterminate potential (CHIP)), technical (e.g., limits of detection and variable levels of tumor fraction (TF) observed in cancer patients), and practical (e.g., blood volume requirements and cost) considerations [10-12]. In patients with cancer, ctDNA generally represents a small fraction of all cfDNA, ranging from $\geq 5-10 \%$ in late-stage disease to $\leq 0.01-1.0 \%$ in early-stage disease, and even lower in premalignant conditions [13]. These limitations are particularly important in early-stage cancer when the tumor is small and the shedding of DNA into the blood may be minimal. Indeed, many previous cfDNA studies have had stage distributions meaningfully different from those seen in screening populations [14-16].

An alternative to detection based solely on ctDNA is to look more broadly at cfDNA-both tumor derived and non-tumor derived-and changes that early-stage cancer may induce in blood. There is growing evidence of interactions between cancerous cells and other cells, including fibroblasts, platelets, and immune cells, especially within the tumor microenvironment. These include findings of "tumor education", such as changes in gene expression that may reflect interaction with a tumor and/or ingestion of tumor-related molecules [17]. For example, platelets in patients with cancer harbor different patterns of messenger RNA (mRNA) than platelets in healthy individuals [18]. There are also reports of changes in immune-cell apoptosis patterns in patients with cancer [19], suggesting global changes in hematopoietic cell populations that may reflect altered physiological states. For instance, low relative levels of circulating lymphocytes versus monocytes may be correlated with poor cancer prognosis [20]. It is possible to detect such changes in cell populations from cfDNA because cfDNA fragmentation and methylation patterns can recapitulate expected cellular epigenetic states [15, 16, 21-23].

Because it is still unknown to what extent circulating cells in patients with early-stage cancer are educated by the tumor microenvironment (i.e., how changes in cellular state are explicitly reflected in the billions of base pairs of cfDNA), the ability to identify disease-relevant patterns in cfDNA requires unbiased methods that can identify patterns in high-dimensional space. Given a large enough sample size, machine learning (ML) may provide a toolset by which to learn disease-related patterns from whole-genome signals directly from patients with and without early-stage cancer. However, the primary challenge in an assay with many measured variables is to identify relevant, low-dimensional features that generalize to the screening population $[24,25]$. As a corollary, it is necessary to mitigate potential confounding variables, defined as variables that are correlated with the clinical label which in this case is the disease label. For example, batch effects or institutional processing effects can be a significant variable that correlates with non-cancerous and cancerous samples.

Here we develop and implement a computational approach for representing and learning associations between cfDNA profiles and cancer status, with a focus on the importance of accounting for known confounding variables. Using this approach, we report classification results for a large cohort of non-cancer controls and early-stage CRC patients.

\section{Methods}

\section{Sample collection}

Human EDTA plasma samples were acquired from 546 patients diagnosed with CRC (Table 1). As controls, plasma samples from 271 unique patients without a current CRC diagnosis were also acquired. In total, 817 de-identified plasma samples were collected from 
Table 1 Clinical characteristics and demographics of CRC patients and non-cancer controls

\begin{tabular}{lllll}
\hline & & CRC & Control & Total Samples \\
& & N =546 & N=271 & N=817 \\
\hline Gender & Female N (\%) & $264(48 \%)$ & $182(67 \%)$ & $446(55 \%)$ \\
& Male N (\%) & $282(52 \%)$ & $84(31 \%)$ & $366(45 \%)$ \\
& Unknown & 0 & $5(2 \%)$ & $5(<1 \%)$ \\
Stage & I & $172(32 \%)$ & N/A & N/A \\
& II & $266(49 \%)$ & & \\
& III & $98(18 \%)$ & & \\
& IV & $6(1 \%)$ & & \\
& Unknown & $4(<1 \%)$ & & \\
Age (yrs) & Median (IQR) & $71(63-80)$ & $60(53-67)$ & $68(59-77)$
\end{tabular}

institutions and commercial biobanks located in the United States, Germany, and Scotland. Patient age, gender, and cancer stage (where available) were obtained for each sample. Samples were included in the intended use (IU) age range analysis for CRC only if the patient's age at time of collection was known to be between 50 and 84 , inclusive. Plasma was stored at $-80^{\circ} \mathrm{C}$.

\section{Laboratory processing, bioinformatics, and featurization}

Detailed descriptions of laboratory processing and sequencing, bioinformatics analysis, data preprocessing, classifier training, and validation methods (including measuring and controlling for confounding factors) are provided in the Additional file 1: Supplemental Methods.

Briefly, cfDNA was extracted from $250 \mu \mathrm{l}$ plasma using the MagMAX cfDNA Isolation Kit (Applied Biosystems), converted into libraries using the NEBNext Ultra II DNA Library Prep Kit (New England Biolabs), and paired-end sequenced on the Illumina platform. Reads were aligned to the human genome using BWA-MEM 0.7.15 [26]; all datasets passing quality control (based on acceptable GC bias, sufficient number of reads, and no evidence of contamination or sample swaps) continued to featurization. Aligned reads were transformed into per-sample feature vectors by counting the number of fragments appearing in protein-coding genes. Features were normalized per-sample by dividing by the trimmed mean (excluding top and bottom 10\% of counts) over all features and applying Loess GC bias correction [27]. Categorical features used in certain experiments (binned age, sex, and institution) were featurized using a one-hot encoding. Tumor fraction was estimated in each sample using IchorCNA [28] from read counts in 50-kilobase $(\mathrm{kb})$ bins across the entire genome. Prior to the authors' method, $50 \mathrm{~kb}$ - bin counts were normalized using tangent normalization with a panel of non-cancer control and process control samples.

\section{Model training}

ML models were trained and evaluated using cross-validation (CV) procedures as follows. Each feature, which is a preprocessed read count, was standardized by subtracting the mean and dividing by the standard deviation after large outliers were replaced with 99th percentile value. Dimension-reduction methods including principal component analysis (PCA) and truncated singular-value decomposition (SVD) were then optionally applied to the standardized data. Two classification methods were considered for training (logistic regression and support vector machine (SVM)) with hyperparameters chosen based on random search within the training data of each fold; the test folds were reserved exclusively for the average test performance estimate described in the following section. The detailed procedure used for model selection is described in the Additional file 1: Supplemental Methods. The best model was selected based on k-fold $\mathrm{CV}$, and the methods were subsequently applied to other $\mathrm{CV}$ procedures. All methods were implemented by Scikit-learn [29].

\section{Validation and confounder control}

We used CV to estimate a classification model's performance on new, previously unseen data. Five different $\mathrm{CV}$ procedures were used to obtain estimates of model performance. All CV procedures shared in common the partitioning of the data into multiple independent subsets, or "folds," with individual folds held out and used to assess the performance of models trained on the remaining data (Fig. 1a). The principal difference among CV procedures was how individual samples were partitioned into folds (Fig. 1b). The procedures included kfold, in which samples were partitioned at random (stratified by class label of cancer or not cancer); binnedage, in which partitions were defined based on age; kbatch, in which partitions were defined by processing batch; balanced k-batch, in which partitions were defined by processing batch with additional downsampling to stratify by institutional source; and ordered k-batch, in which samples were partitioned by date of laboratory processing. Details of each method are provided in the Additional file 1: Supplemental Methods.

Five folds $(k=5)$ were used for $\mathrm{CV}$ of all models except for binned-age (which has a fixed number of bins). Reported performance metrics are mean area under the curve (AUC), where curve is the receiver operating characteristic (ROC) curve, and mean sensitivity at $85 \%$ specificity, each calculated across all test folds. 

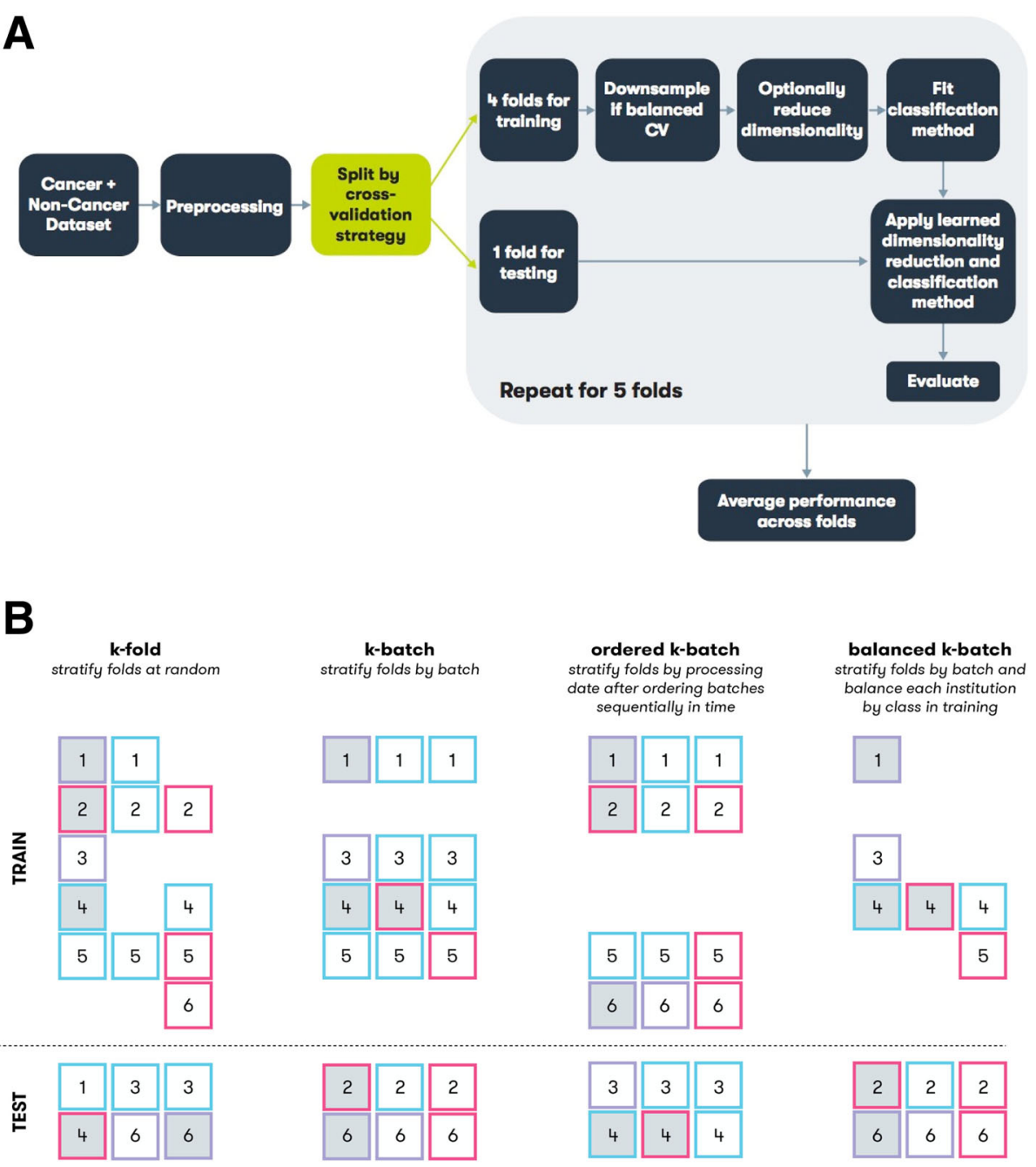

Non-cancer control / cancer

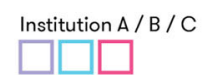

Processing batch \#

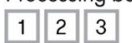

Fig. 1 Model training overview and CV procedures. a All methods were trained on k-fold, and the best performing method was chosen to train models for the other cross-validation procedures. Diagram describes individual steps in common to all methods. Models are trained on a given dataset and set of methods (i.e., dimension reduction and classification) and then evaluated, resulting in a performance estimate. $\mathbf{b}$ Illustration of $\mathrm{CV}$ procedures for $\mathrm{k}$-fold, k-batch, ordered k-batch, and balanced k-batch. Each square represents a single sample, with the fill color indicating class label, the border color representing a confounding factor like institution, and the number indicating processing batch. Each column represents a possible fold constructed for the given CV procedure. The dashed line separates the test set of samples held out from the training set

\section{Results}

Paired-end whole-genome sequencing (WGS) was performed on plasma cfDNA obtained from 271 non-cancer control subjects and 546 CRC patients (Table 1). The patient population was approximately equally split by gender (55\% female, $45 \%$ male), and consisted of $80 \%$ early-stage (stages I and II) patients. The non-cancer control population skewed younger (median age $=60$; interquartile range $[\mathrm{IQR}]=53-67)$ than the cancer population (median age = 71; IQR $=63-80, p<0.01$, Mann-Whitney U-test) (Table 1).
WGS data were converted into input features for the classification model by counting the number of fragments overlapping each annotated protein-coding gene (i.e., each gene corresponded to a single bin) and then normalizing to account for feature length, mappability, read depth, and sequence-content biases. The genebased featurization was designed to simultaneously capture both copy number changes as well as epigenetic signals reflected in cfDNA fragmentation patterns across genes [15]. 


\section{Performance of confounder covariates}

Before assessing classification performance, models were trained using confounding variables as inputs to validate our CV stratification methods. In k-fold CV, binned age, batch, processing date, and institution confounders achieved mean AUCs of 0.71, 0.72, 0.69, and 0.87, respectively, when tested individually as the only input features to the classification model (Table 2, Additional file 2: Figure S1). When evaluated using CV methods tailored specifically to address them, these same input features (i.e., confounder variables) had no predictive power (i.e., AUCs of approximately 0.50). These results demonstrate that binned-age, k-batch, balanced k-batch, and ordered k-batch CV effectively assess performance while controlling for their respective confounder variables (Table 2, Additional file 2: Figure S1).

\section{Performance by cross-validation procedures}

After initial model selection via $\mathrm{k}$-fold $\mathrm{CV}$ performance, we additionally applied each previously introduced CV procedure to the same methods to estimate the generalizability of performance when controlling for particular confounder variables individually (Table 3 ). The method selected by $\mathrm{k}$-fold $\mathrm{CV}$ performance was no dimensionality reduction and SVM classification. Evaluation by standard k-fold CV achieved a mean AUC of 0.92 (95\% bootstrap confidence interval (CI) of 0.910.93 ) with a mean sensitivity of $85 \%(95 \% \mathrm{CI}=83-86 \%)$ at $85 \%$ nominal specificity. Using binned-age CV to control for age achieved mean AUC of $0.91(95 \% \mathrm{CI}=0.89$ $0.94)$ with a mean sensitivity of $79 \%(95 \% \mathrm{CI}=73-87 \%)$ at $85 \%$ specificity. We controlled batch-to-batch technical variability using $k$-batch $\mathrm{CV}$ and process variability using ordered k-batch $\mathrm{CV}$, which achieved mean AUC of $0.91(95 \% \mathrm{CI}=0.88-0.94)$ and $0.90(95 \% \mathrm{CI}=0.83-$ $0.94)$ and sensitivity at $85 \%$ specificity of $85 \%(95 \% \mathrm{CI}=$ $80-89 \%)$ and $73 \%(95 \% \mathrm{CI}=53-88 \%)$, respectively. The larger variance observed in ordered k-batch may be attributed (at least in part) to higher standard deviation in test fold sizes (80.8) when compared to standard deviation of test folds of $\mathrm{k}$-batch (35.0) (Additional file 6: Table S1). Finally, we applied balanced k-batch CV to control for possible institution-specific differences in population or sample handling. Despite training on a significantly reduced dataset (average of 263. 6 samples per fold in training versus 653. 6 samples per fold with $\mathrm{k}$-fold or k-batch as seen in Additional file 6: Table S1), the balanced k-batch CRC model achieved a mean AUC of $0.83(95 \% \mathrm{CI}=0.79-0.86)$ with a mean sensitivity of $71 \%(95 \% \mathrm{CI}=63-76 \%)$ at $85 \%$ specificity (Table 3 ). Figure 2 shows ROC curves for each $\mathrm{CV}$ procedure.

\section{Detailed comparison of performance by clinical parameters}

Additionally, we investigated the sensitivity of our method, trained using each $\mathrm{CV}$ procedure, to relevant clinical parameters. Figure 3a illustrates sensitivity as a function of clinical stage. All validation methods achieved similar distributions of sensitivity across stages I through III, and consistently classified stage IV cancer correctly. Stage II samples, which represent the majority of our data, performed consistently well. We also evaluated age, which is a known confounder. The AUC performance increased with age in nearly all validations (Fig. 3b). Taken as a whole, the results are consistent with the general notion that cancer is an age-related disease. Performance for males and females was comparable across validation types (Fig. 3c), even in spite of the observed imbalance in non-cancer controls (Table 1).

Tumor fraction (TF), defined as the fraction of cfDNA originating from tumor cells, has been implicated as a critical parameter for the design of blood-based cancer screens $[4,6,12,30,31]$. As high-depth mutation detection information is not available in our data, we estimated TF from observed copy number variation using IchorCNA [28]. Nearly all of the non-cancer control samples (>99\%) were estimated to have a TF below $0.8 \%$ (Additional file 3: Figure S2).

Figure 3d displays our CRC model's AUC as a function of an IchorCNA-based estimated TF. Observed performance increased with increasing TF, which is consistent with the hypothesis that an ML-based method may be able to detect tumor-derived signal; however, performance remained better than chance even in the lowest TF bin. To investigate whether the ML model may detect signal beyond tumor-derived CNVs, we used an IchorCNA-based estimated TF alone to predict cancer. This method achieved AUC of 0.67 in the IU age range,

Table 2 Performance Evaluation of Known Confounders

\begin{tabular}{lllll}
\hline Confounder & k-fold CV AUC (95\% Cl) & k-fold CV Sensitivity at 85\% Specificity (95\% Cl) & Confounder CV method & Confounder CV AUC (95\% Cl) \\
\hline Age & $0.71(0.64-0.77)$ & $44 \%(29-57 \%)$ & Binned-age & $0.50(0.50-0.50)$ \\
Batch & $0.72(0.69-0.75)$ & $43 \%(31-53 \%)$ & k-batch & $0.50(0.50-0.50)$ \\
Processing Date & $0.69(0.64-0.74)$ & $38 \%(25-49 \%)$ & Ordered k-batch & $0.48(0.43-0.52)$ \\
Institution & $0.87(0.84-0.90)$ & $74 \%(72-77 \%)$ & Balanced k-batch & $0.51(0.28-0.74)$ \\
\hline
\end{tabular}

Performance evaluation of known confounders alone to predict cancer with either k-fold or the CV procedure designed to control for the confounder. Confidence intervals are calculated from bootstrapped distributions of the metric across folds 
Table 3 CRC Performance by Validation Procedure

\begin{tabular}{lll}
\hline Validation & $\begin{array}{l}\text { Mean AUC } \\
(95 \% \mathrm{Cl})\end{array}$ & $\begin{array}{l}\text { Mean Sensitivity at } \\
85 \% \text { Specificity }(95 \% \mathrm{Cl})\end{array}$ \\
\hline k-fold & $0.92(0.91-0.93)$ & $85 \%(83-86 \%)$ \\
Binned-age & $0.91(0.89-0.94)$ & $79 \%(73-87 \%)$ \\
k-batch & $0.91(0.88-0.94)$ & $85 \%(80-89 \%)$ \\
Ordered k-batch & $0.90(0.83-0.94)$ & $73 \%(53-88 \%)$ \\
Balanced k-batch & $0.83(0.79-0.86)$ & $71 \%(63-76 \%)$ \\
\hline
\end{tabular}

CRC performance by cross-validation procedure in 50-84 year-old patients. Confidence intervals are calculated from bootstrapped distributions of the metric across folds

lower than results from the ML model under any analyzed CV scheme (Table 3), consistent with the possibility that the ML model used non-tumor-derived signal (i.e., beyond IchorCNA-detectable $\mathrm{CNVs}$ ) (Additional file 4: Figure S3).

To address decreased classifier performance due to smaller sample sizes in training (i.e., balanced k-batch), the CRC dataset was downsampled. Additional file 5: Figure S4 illustrates the non-linear relationship between the total number of samples used for training and the measured sensitivity. These results suggest that the lower performance observed using balanced k-batch is explained, at least in part, by the smaller size of the training dataset.

\section{Discussion}

Our results show promising preliminary performance for early-stage (i.e., stages I and II) CRC detection using blood. To our knowledge, this multicenter, international study represents the largest study to date using only cfDNA WGS in patients for the early detection of CRC. We have demonstrated that it is possible to take an MLbased approach to learn the relationship between a patient's cfDNA profile and cancer diagnosis, with $85 \%$ sensitivity at $85 \%$ specificity in CRC using standard kfold cross-validation; application of other rigorous and novel CV strategies specifically designed to control for known confounding variables yielded $71-85 \%$ sensitivity at $85 \%$ specificity.

In this work, we focused our approach on cfDNA count profiles across the whole human genome $(\sim 3200$ $\mathrm{Mb})$ at relatively low depth $(\sim 9 \mathrm{X})$, as opposed to existing liquid biopsy approaches that assess small regions $(<2$ $\mathrm{Mb})$ of the genome at very high depth $(\sim 60,000 \mathrm{X})$ to detect tumor-derived mutations. In particular, we applied ML methods to perform unbiased discovery of signals of varying origin that may inform on the presence of a tumor (including both tumor-derived CNVs as well as potentially non-tumor-derived signals such as changes in the epigenetic states of circulating immune cells) visà-vis focusing on only tumor-derived mutations. This parallels previous research in non-invasive prenatal testing (NIPT): Kim et al. demonstrated that an MLbased regression algorithm operating on genome-wide count data was able to accurately estimate fetal fraction in the cfDNA of pregnant women, without the detection of single-nucleotide polymorphism differences between mother and fetus [32]. Additionally, unlike liquid biopsy approaches using ultra-high-depth sequencing, the use of relatively low depth meaningfully decreases the cost of testing and permits the use of reasonable blood volumes, both of which will ultimately be required for population-level screening [12]. Finally, approaches focused on mutation detection alone can miss certain types of tumor-derived signals (e.g., genome-wide CNVs and epigenetic modifications), which are by definition most scarce in early-stage (i.e., non-metastatic) disease and pre-cancerous lesions, the detection of which is the goal of cancer screening programs.

While we have not yet directly determined the exact contributions to classifier performance from tumor- versus non-tumor-derived sources, several lines of evidence suggest that both may be present. First, while the observed relationship between AUC and inferred TF (Fig. 3d) indicates that at least some of the classification power is likely attributable to the ability of the model to identify samples with abundant ctDNA, the ability to correctly classify samples with lower TF and/or earlystage disease suggests that ctDNA alone cannot fully account for classification performance. Second, a CRC classification model based solely on an estimated TF (inferred from CNV calls) performs relatively poorly, with an AUC lower than all tested CV results for the ML method, suggesting that non-CNV sources may contribute to our ML-based classifier. Future research will focus on better understanding the underlying biology of the classifier, as well as assessing potential improvements in model performance from the addition of other analytes and ML method development, including confounder mitigation.

In the presence of inadequately controlled confounders, ML methods are prone to learn irrelevant associations; this poses a critical challenge for the use of ML for biomarker discovery [33-36]. Certain confounders can be mitigated "up front" through experimental design (e.g., demographic biases and institution bias) or operational quality control (e.g., identification of known parameter drift). This can help minimize the dependence between class label and any potential noise-inducing variable but incurs an additional cost in time and/or operational expense. However, perfect control of confounders at the design stage is not realistic: Some variables may be intrinsically confounding in the population of interest (e.g., cancer incidence increases with age), and there are modes of variation which may exist but which may not be known a priori and therefore 

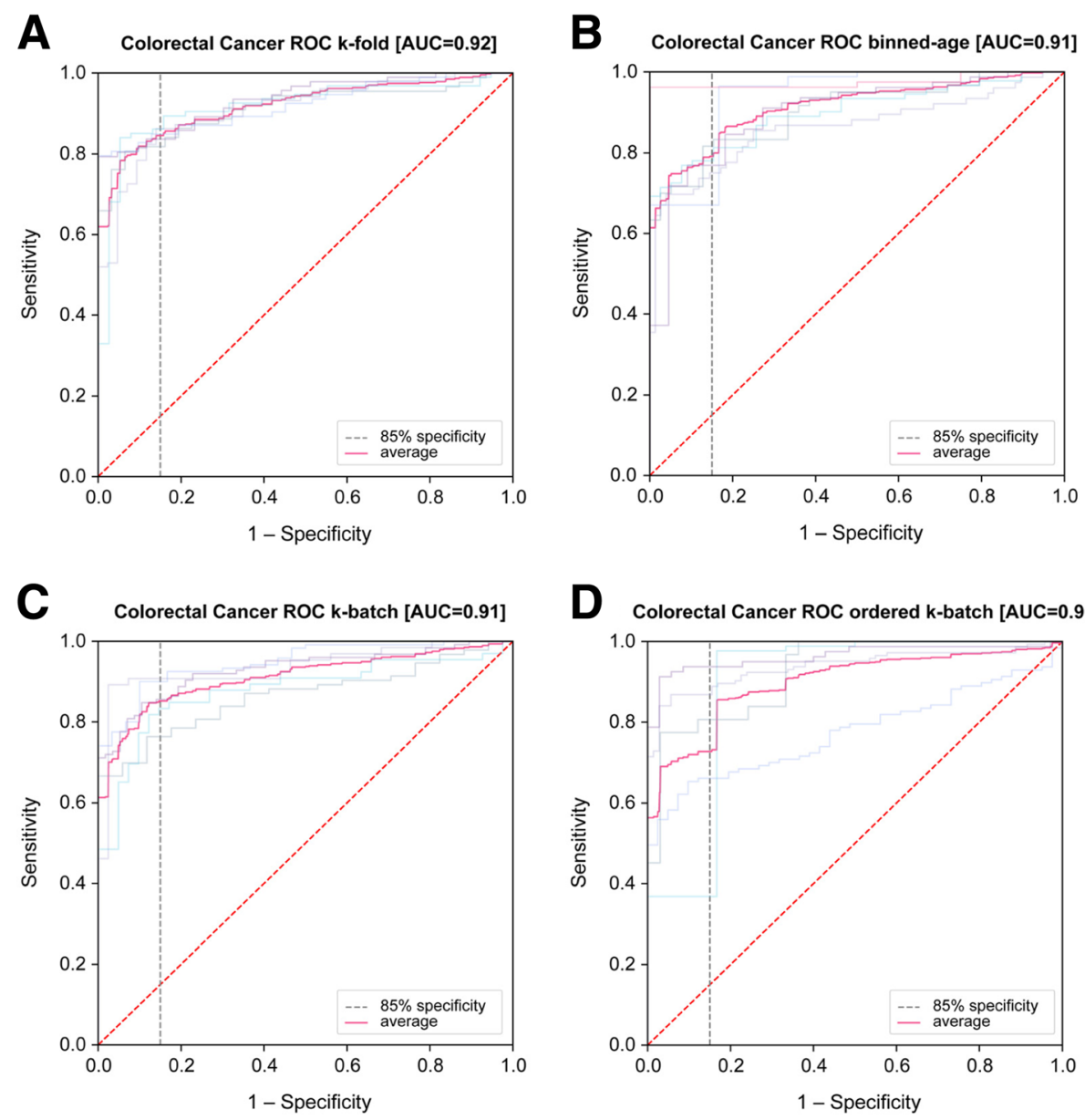

D Colorectal Cancer ROC ordered $k$-batch [AUC $=0.90$

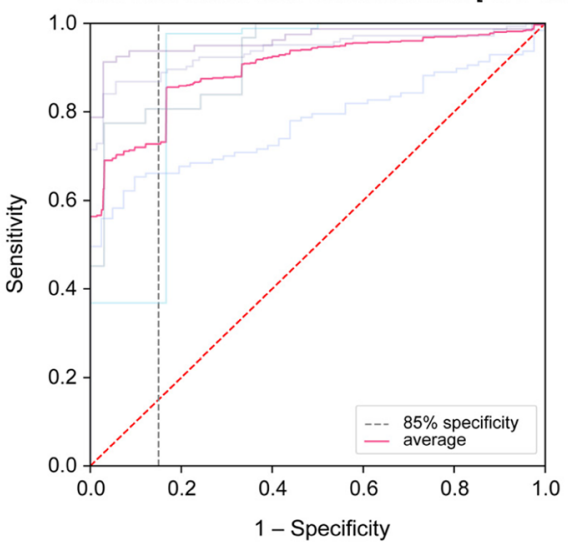

E Colorectal Cancer ROC balanced k-batch [AUC $=0.83]$

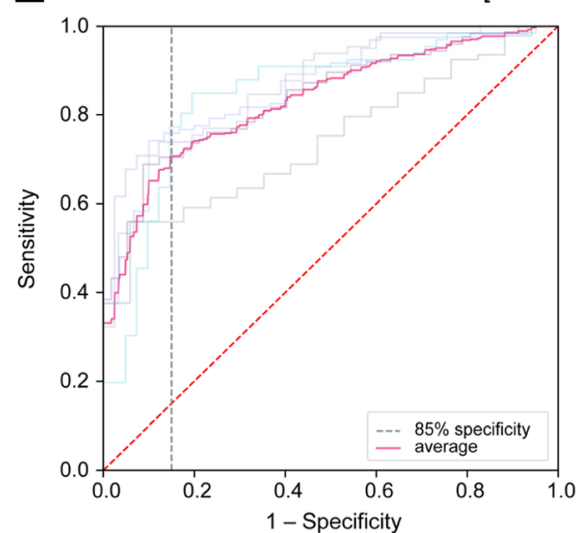

Fig. 2 Colorectal cancer classification performance (ROC curves) by each cross-validation method. Average of all folds drawn in solid blue; random chance is represented as dashed red; ROCs for each fold drawn behind. $\mathbf{a}$ k-fold, $\mathbf{b}$ binned age, $\mathbf{c}$ k-batch, $\mathbf{d}$ ordered k-batch, and $\mathbf{e}$ balanced k-batch

mitigated post hoc (e.g., batch-to-batch variability in sequencing).

A key contribution of this work is the presentation and analysis of cross-validation techniques specifically tailored to go beyond traditional $\mathrm{k}$-fold validation to measure and mitigate a number of pervasive confounding effects in biomarker discovery: k-batch and ordered k-batch for different scales of process 

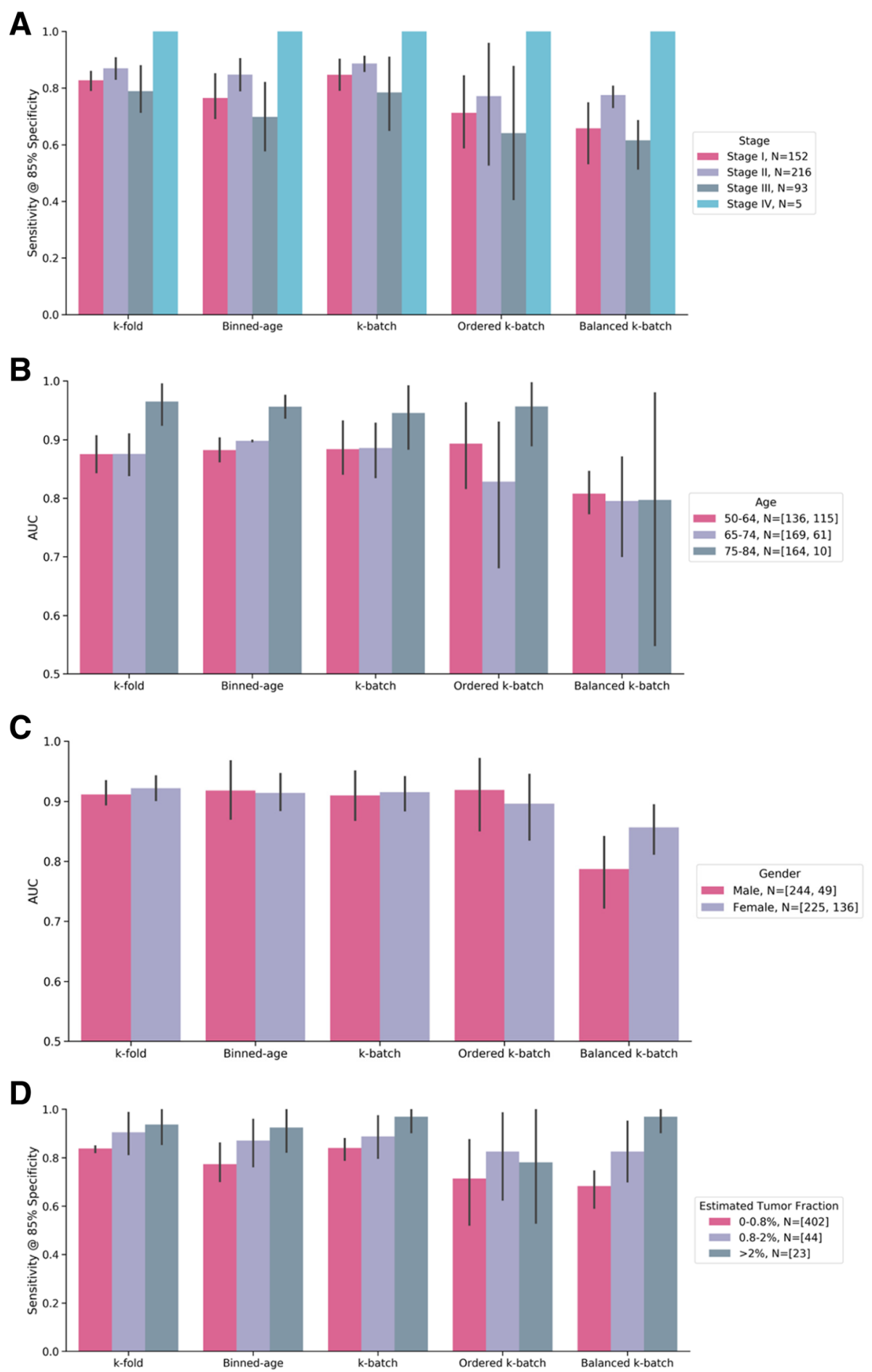

Fig. 3 Classification performance for colorectal cancer within the IU age range across all validation methods. $\mathrm{N}$ is number of samples, [cancer, controls]. The average of all folds is represented by the colored bars; the $95 \%$ bootstrap confidence intervals are represented by the solid black lines. a Sensitivity at $85 \%$ nominal specificity by CRC stage across all CV procedures. b AUC by age bins across all CV procedures. c AUC by gender across all CV procedures. d AUC by an IchorCNA-based estimated TF across all CV procedures

variability in time, respectively, and balanced k-batch for institution-specific biases. We found that standard $\mathrm{k}$-fold CV can have higher performance than confounder-controlled CV methods, consistent with the historical difficulty in reproducing discovery studies. We believe that explicit stratification for technical 
and biological confounders may be used as standard practice to better evaluate the generalizability of early discovery results.

The current study has a number of potential limitations. First, because samples were obtained retrospectively, breaks in the chain of custody may have led to sample and labelling errors, which would impede the ability of an ML method to adequately learn. Additionally, the presence of CNVs in a small number of control samples (Additional file 3: Figure S2) has been previously observed in other cohorts and may be due to malignant or benign causes [ 5 , 14, 37]; further follow up was not possible in this cohort. Another limitation of our study is that TF was estimated using copy-number inference from moderate-coverage whole-genome sequencing, which as implemented here has a limit of detection of $0.8 \%$ for TF; by contrast, targeted mutation detection would allow more sensitive characterization of TF. Finally, cross validation is a widely used approach for assessment of classification performance and, as implemented here, can address known individual confounders. However, cross-validation procedures do not control for all confounders simultaneously, and the use of an independent test set is ultimately needed to evaluate the generalizability of these results. Prospective studies are underway to validate classifier performance in an independent cohort and verify generalization predictions from confoundercontrolled CV.

Although this study focused on CRC, this study approach is directly applicable to other cancers and indeed to other pathological and physiological conditions. Our approach extracts signals from certain biological states and can apply them to better understand others; however, full development and validation of classifiers to address different clinical and non-clinical applications will require additional samples in those specific populations. Unlike targeted mutation approaches which require identification of disease-specific targets, this whole genome approach allows for the unbiased discovery of signals which are not disease-specific and could even be extended to the assessment and monitoring of non-disease states. Additionally, this approach should be able to detect unique epigenetic patterns for other diseases, thereby providing specificity by differentiating CRC from other cancers [38]. Efforts are currently underway to evaluate these hypotheses.

\section{Conclusions}

In summary, this study presents a novel representation of cfDNA and an analysis framework that demonstrates promising initial results for the detection of early-stage CRC based on a minimally invasive blood test. Prospective validation of this approach is currently underway, as is the incorporation of other cellfree, blood-based analytes (e.g., proteins) that may contribute orthogonal signals to further improve classifier performance.

\section{Additional files}

\begin{abstract}
Additional file 1: Supplemental Methods. (DOCX $26 \mathrm{~kb}$ )
Additional file 2: Figure S1. Performance of confounding variable in kfold and target CV procedure (ROC curves) for: a) Age and binned-age CV b) Batch and k-batch CV c) Processing date and ordered k-batch CV d) Institution and balanced k-batch CV. Note that stratification CVs (i.e., binned-age, k-batch, and ordered k-batch) perform at chance (mean AUC of 0.50 ) when the distribution of labels in the test covariate is even and would result in an $\mathrm{ROC}$ that is just a diagonal line. The variance seen in some of the target procedures is due to class imbalance in the test data. (DOCX 420 kb)
\end{abstract}

Additional file 3: Figure S2. Percentage of colorectal cancer samples by stage and non-cancer controls in each range of estimated TF (based on observed CNV using an IchorCNA-based estimate). (DOCX 74 kb)

Additional file 4: Figure S3. IchorCNA-based estimated TF alone to predict case status (i.e., cancer or control) achieved an AUC of 0.67 in the $\mathrm{IU}$ age range, meaningfully lower than results from the $\mathrm{ML}$ model under any analyzed CV procedure. (DOCX 101 kb)

Additional file 5: Figure S4. Non-linear relationship between the total number of samples used for training and sensitivity at $85 \%$ specificity for colorectal cancer detection. The method was trained again with k-fold, except the number of training samples per fold was downsampled. The lower numbers are comparable to those available for balanced k-batch and were used to investigate decreased classifier performance due to smaller sample sizes in training. (DOCX $60 \mathrm{~kb}$ )

Additional file 6: Table S1. Mean and standard deviation of the number of samples (both in and out of the IU age range) per fold for each CV procedure. SD = standard deviation. (DOCX $16 \mathrm{~kb}$ )

\section{Abbreviations}

AUC: Area under the curve, where curve is the receiver operating characteristic; cfDNA: Cell-free DNA; CHIP: Clonal hematopoiesis of indeterminate potential; CNV: Copy number variant; ctDNA: Circulating tumor DNA; CV: Cross-validation; DNA: Deoxyribonucleic acid; IU: Intended use; ML: Machine learning; RNA: Ribonucleic acid; ROC: Receiver operating characteristic; SVM: Support vector machine; TF: Tumor fraction

\section{Acknowledgements \\ The authors gratefully acknowledge Dr. Samuel So from the Asian Liver Center at Stanford University, Dr. Randall Brand from the University of Pittsburgh Medical Center, Dr. Andrew Godwin and the Biospecimen Repository Core Facility staff funded in part by the National Cancer Institute Cancer Center Support Grant (P30 CA168524), National Health Services Research Scotland, Tayside Biorepository, Geneticist Inc., iSpecimen Inc., and Indivumed for support of this research by providing de-identified plasma samples.}

\section{Authors' contributions}

NW and DW led analysis and writing of the manuscript. NW, MB, NB, AD, EG, LH, JSJ, AK, TL, YL, KN, GES, BW, and ISH developed and applied computational analysis techniques. DW, EA, JC, and DD developed lab protocols and automation for sample analysis. $E A, M B, D B, J P, B R, A S, C T, A T$, $L Y$, and GP performed data acquisition and verification, including sample processing and clinical metadata verification. EA, LH, TL, KN, SF, GP, and ISH made significant contributions to drafting the manuscript. ISH, GP, GO, and RE supervised the research. All authors read and approved the final manuscript. 


\section{Funding}

Not applicable

\section{Availability of data and materials}

Underlying raw sequencing data are not available due to privacy protection laws.

\section{Ethics approval and consent to participate}

Quorum Review Inc. (Seattle, WA) provided a human subject exemption letter covering this research. (reference QR \#: 33472). Specifically, after review of the study it was deemed that the described activity does not constitute research involving human subjects as defined in 45 CFR \$46.102. The study activities did not involve the collection of data through intervention or interaction with the individual because the activity utilized plasma samples collected from commercial biobanks and from other academic clinical studies. Furthermore, no identifiable private information was obtained because the aforementioned plasma samples were de-identified prior to receipt and no there is no access to the identifiers. Therefore, the previously conducted study activities did not constitute research involving human subjects.

\section{Consent for publication}

Not applicable

\section{Competing interests}

$N W, D W, T L, E A, M B, M B, D B, J C, A D, R E, S F, L H, J S J, G O, J P, B R, G E S, A S, C T$, and $L Y$ are employees of Freenome.

GP is an employee of Freenome and independent contractor/consultant for Palmetto GBA, MolDX

$\mathrm{KN}, \mathrm{AK}, \mathrm{DD}, \mathrm{BW}, \mathrm{NB}, \mathrm{EG}, \mathrm{AT}, \mathrm{YL}$ and ISH are former employees of Freenome. Freenome is the sponsor of NCT03688906 (Al-EMERGE: Development and Validation of a Multi-analyte, Blood-based Colorectal Cancer Screening Test).

Received: 10 February 2019 Accepted: 31 July 2019

Published online: 23 August 2019

\section{References}

1. Shapiro JA, Klabunde CN, Thompson TD, Nadel MR, Seeff LC, White A. Patterns of colorectal cancer test use, including $C T$ colonography, in the 2010 National Health Interview Survey. Cancer Epidemiol Biomarkers Prev. 2012;21:895-904

2. Siegel RL, Miller KD, Jemal A. Cancer statistics, 2018. CA Cancer J Clin. 2018;68:7-30.

3. Colonoscopy for Colorectal Cancer Screening. [cited 2018 Nov 15]. Available from: http://www.jcancer.org/v04p0217.htm

4. Bettegowda C, Sausen M, Leary RJ, Kinde I, Wang Y, Agrawal N, et al. Detection of circulating tumor DNA in early- and late-stage human malignancies. Sci Transl Med. 2014;6:224ra24.

5. Aravanis AM, Lee M, Klausner RD. Next-Generation Sequencing of Circulating Tumor DNA for Early Cancer Detection. Cell. 2017;168:571-4.

6. Phallen J, Sausen M, Adleff V, Leal A, Hruban C, White J, et al. Direct detection of early-stage cancers using circulating tumor DNA. Sci Transl Med. 2017;9:eaan2415.

7. Cohen JD, Li L, Wang Y, Thoburn C, Afsari B, Danilova L, et al. Detection and localization of surgically resectable cancers with a multi-analyte blood test. Science. 2018;359:926-30.

8. Heitzer E, Ulz P, Geigl JB. Circulating tumor DNA as a liquid biopsy for cancer. Clin Chem. 2015;61:112-23.

9. Jiang P, Lo YMD. The Long and Short of Circulating Cell-Free DNA and the Ins and Outs of Molecular Diagnostics. Trends Genet. 2016:32:360-71.

10. Heitzer E, Haque IS, Roberts CES, Speicher MR. Current and future perspectives of liquid biopsies in genomics-driven oncology. Nat Rev Genet. 2018;20(2):71-88

11. Heitzer E, Perakis S, Geigl JB, Speicher MR. The potential of liquid biopsies for the early detection of cancer. NPJ Precis Oncol. 2017:1:36.

12. Haque IS, Elemento O. Challenges in Using ctDNA to Achieve Early Detection of Cancer. bioRxiv. 2017:237578

13. Wan JCM, Massie C, Garcia-Corbacho J, Mouliere F, Brenton JD, Caldas C, et al. Liquid biopsies come of age: towards implementation of circulating tumour DNA. Nat Rev Cancer. 2017;17:223-38.

14. Klein EA, Hubbell E, Maddala T, Aravanis A, Beausang JF, Filippova D, et al. Development of a comprehensive cell-free DNA (cfDNA) assay for early detection of multiple tumor types: The Circulating Cell-free Genome Atlas (CCGA) study. J Clin Orthod Am Soc Clin Oncol. 2018:36:-12021.

15. Snyder MW, Kircher M, Hill AJ, Daza RM, Shendure J. Cell-free DNA Comprises an In Vivo Nucleosome Footprint that Informs Its Tissues-OfOrigin. Cell. 2016;164:57-68.

16. Kang S, Li Q, Chen Q, Zhou Y, Park S, Lee G, et al. CancerLocator: noninvasive cancer diagnosis and tissue-of-origin prediction using methylation profiles of cell-free DNA. Genome Biol. 2017;18:53.

17. Pollard JW. Tumour-educated macrophages promote tumour progression and metastasis. Nat Rev Cancer. 2004;4:71-8.

18. Best MG, Sol N, Kooi I, Tannous J, Westerman BA, Rustenburg F, et al. RNA-Seq of Tumor-Educated Platelets Enables Blood-Based Pan-Cancer, Multiclass, and Molecular Pathway Cancer Diagnostics. Cancer Cell. 2015;28:666-76.

19. Whiteside TL. Apoptosis of immune cells in the tumor microenvironment and peripheral circulation of patients with cancer: implications for immunotherapy. Vaccine. 2002;20(Suppl 4):A46-51.

20. Wu Q, Hu T, Zheng E, Deng X, Wang Z. Prognostic role of the lymphocyteto-monocyte ratio in colorectal cancer: An up-to-date meta-analysis. Medicine. 2017:96:e7051.

21. Chan KCA, Jiang P, Chan CWM, Sun K, Wong J, Hui EP, et al. Noninvasive detection of cancer-associated genome-wide hypomethylation and copy number aberrations by plasma DNA bisulfite sequencing. Proc Natl Acad Sci U S A. 2013;110:18761-8.

22. Jiang $\mathrm{P}$, Sun $\mathrm{K}$, Tong $\mathrm{YK}$, Cheng SH, Cheng THT, Heung MMS, et al. Preferred end coordinates and somatic variants as signatures of circulating tumor DNA associated with hepatocellular carcinoma. Proc Natl Acad Sci U S A. 2018;115(46):E10925-33.

23. Ulz P, Thallinger GG, Auer M, Graf R, Kashofer K, Jahn SW, et al. Inferring expressed genes by whole-genome sequencing of plasma DNA. Nat Genet. 2016:48:1273.

24. Sima C, Dougherty ER. The peaking phenomenon in the presence of feature-selection. Pattern Recognit Lett. 2008;29:1667-74.

25. Hua J, Tembe WD, Dougherty ER. Performance of feature-selection methods in the classification of high-dimension data. Pattern Recognit. 2009;42:409-24.

26. Li H. Aligning sequence reads, clone sequences and assembly contigs with BWA-MEM. arXiv [q-bio.GN]. 2013.

27. Cleveland WS. Robust Locally Weighted Regression and Smoothing Scatterplots. J Am Stat Assoc. 1979;74:829-36.

28. Adalsteinsson VA, Ha G, Freeman SS, Choudhury AD, Stover DG, Parsons HA, et al. Scalable whole-exome sequencing of cell-free DNA reveals high concordance with metastatic tumors. Nat Commun. 2017:8:1324.

29. Pedregosa F, Varoquaux G, Gramfort A, Michel V, Thirion B, Grisel O, et al. Scikitlearn: Machine Learning in Python. J Mach Learn Res. 2011;12:2825-30.

30. Delubac D, Ariazi E, Berliner J, Drake A, Dulin J, Ennis R, et al. Abstract 2227: Multi-analyte profiling reveals relationships among circulating biomarkers in colorectal cancer. Cancer Res Am Assoc Cancer Res. 2018;78:2227.

31. Newman AM, Bratman SV, To J, Wynne JF, Eclov NCW, Modlin LA, et al. An ultrasensitive method for quantitating circulating tumor DNA with broad patient coverage. Nat Med. 2014;20:548-54.

32. Kim SK, Hannum G, Geis J, Tynan J, Hogg G, Zhao C, et al. Determination of fetal DNA fraction from the plasma of pregnant women using sequence read counts. Prenat Diagn. 2015;35:810-5.

33. Tinker AV, Boussioutas A, Bowtell DDL. The challenges of gene expression microarrays for the study of human cancer. Cancer Cell. 2006;9:333-9.

34. Ransohoff DF, Gourlay ML. Sources of bias in specimens for research about molecular markers for cancer. J Clin Oncol. 2010;28:698-704.

35. Leek JT, Scharpf RB, Bravo HC, Simcha D, Langmead B, Johnson WE, et al. Tackling the widespread and critical impact of batch effects in highthroughput data. Nat Rev Genet. 2010;11:733-9.

36. Goh WWB, Wang W, Wong L. Why Batch Effects Matter in Omics Data, and How to Avoid Them. Trends Biotechnol. 2017:35:498-507.

37. Osborne CM, Hardisty E, Devers P, Kaiser-Rogers K, Hayden MA, Goodnight W, et al. Discordant noninvasive prenatal testing results in a patient subsequently diagnosed with metastatic disease. Prenat Diagn. 2013;33:609-11.

38. Corces MR, Granja JM, Shams S, Louie BH, Seoane JA, Zhou W, et al. The chromatin accessibility landscape of primary human cancers. Science. 2018;362:aav1898.

\section{Publisher's Note}

Springer Nature remains neutral with regard to jurisdictional claims in published maps and institutional affiliations. 